\title{
Long-Term Outcomes of Pulmonary Resection for Lung Cancer Patients with Chronic Kidney Disease
}

\author{
Yoko Yamamoto $^{1} \cdot$ Ryu Kanzaki $^{1,2} \cdot$ Takashi Kanou $^{1} \cdot$ Naoko Ose $^{1} \cdot$ Soichiro Funaki $^{1}$. \\ Masato Minami $^{1} \cdot$ Yasushi Shintani $^{1}$
}

Published online: 4 September 2019

(C) The Author(s) 2019

\begin{abstract}
Background The survival outcome in lung cancer patients with chronic kidney disease (CKD) has not been well evaluated. The aim of this study was to evaluate the survival outcomes following non-small cell lung cancer (NSCLC) surgery in patients with CKD as a preoperative comorbidity.

Methods Among 671 patients who underwent surgery for NSCLC between 2007 and 2014 at our hospital, 55 (8\%) had CKD and we retrospectively analyzed the survival outcomes of these patients.

Results Most patients with CKD were elderly and male. Patients with CKD had a higher frequency of smoking habit, cardiovascular disease, and pulmonary diseases, and a notably lower pulmonary function, resulting in receiving limited pulmonary resection. There were no marked differences in the frequency of surgical complications between patients with and without CKD $(p=0.16)$. Squamous cell carcinoma was more frequently diagnosed in patients with CKD than in those without it. The 5-year disease-free survival rates in patients with and without CKD were $60.0 \%$ and $69.7 \%$ ( $p=0.06$ ), respectively, and the 5-year overall survival rates were $68.9 \%$ and $80.0 \%$, respectively, showing significant differences $(p=0.01)$. The rate of receiving supportive care was higher in patients with CKD when recurrence observed. Conclusion CKD is associated with a poorer overall survival in patients who undergo lung cancer resection for recurrent disease. As patients with CKD tend to have a poor respiratory function, thoracic surgeons should carefully select the resection type to balance the therapeutic benefit and invasiveness.
\end{abstract}

\section{Introduction}

Lung cancer is the leading cause of cancer-related death in the western world [1]. Pulmonary resection is the treatment modality with the best chance of curing early-stage lung cancer. As such, in recent decades, the life expectancy of

Ryu Kanzaki

rkanzaki@tj8.so-net.ne.jp

1 Department of General Thoracic Surgery, Osaka University Graduate School of Medicine, L5-2-2 Yamadaoka, Suita-City, Osaka 565-0871, Japan

2 Experimental Oncology, Division of Translational Cancer Research, Department of Laboratory Medicine, Lund University, 404:A3 Scheelevägen 2, 22381 Lund, Sweden these patients has been increasing, which in turn results in an increasing frequency of a wide range of morbidities such as cardiac and respiratory diseases [2, 3].

Chronic kidney disease (CKD) is a concept that includes all chronic kidney damage or dysfunction, regardless of the primary disease, and was proposed by the National Kidney Foundation in the USA in 2002. CKD is defined as a persistent decline in the renal function to a glomerular filtration rate (GFR) of $<60 \mathrm{ml} / \mathrm{min} / 1.73 \mathrm{~m}^{2}$ or findings that suggest renal impairment for $\geq 3$ months [4]. Several studies have reported that patients with CKD experience a high incidence of malignant tumors [5, 6]. Furthermore, comorbidity of CKD is associated with an increase in surgical complications and the surgical mortality rate, as 
well as a reduced overall survival (OS) in several malignancies [7-11].

Whether or not CKD negatively affects the survival of patients with lung cancer remains controversial. There have been a few reports concerning patients with CKD who underwent medical treatment of lung cancer based on data from a national database [12]. However, to our knowledge, there have been no reports on the outcomes of lung cancer patients with CKD limited to surgical cases. In addition, the influence of CKD on the feasibility of surgery is also unclear, necessitating the clarification of the effects of CKD on patients who undergo surgery.

In the present study, we investigated the impact of CKD on the perioperative and long-term outcomes of pulmonary resection for lung cancer patients.

\section{Patients and methods}

From January 2007 to December 2014, 694 patients underwent pulmonary resection for NSCLC with curative intent at Osaka University Hospital. Patients who received preoperative chemotherapy or radiotherapy were excluded from this retrospective study $(n=23)$, leaving 671 patients enrolled. The study protocol was approved by the Ethical Review Board for Clinical Studies at Osaka University (control number 18237).

Staging was assessed according to the International Association for the Study of Lung Cancer Staging Manual in Thoracic Oncology 7th edition [13]. CKD was defined according to the Clinical Practice Guideline book for the diagnosis and treatment of CKD, 2012, published by the Japanese Society of Nephrology [14]. The renal function was evaluated as the estimated eGFR, calculated as follows: eGFR $\left(\mathrm{ml} / \mathrm{min} / 1.73 \mathrm{~m}^{2}\right)=194 \times$ serum creatinine $(\mathrm{mg} / \mathrm{dl})^{-1.094} \times \mathrm{age}^{-0.278}$ (if female $\times 0.739$ ). CKD was defined as an eGFR $<60 \mathrm{ml} / \mathrm{min} / 1.73 \mathrm{~m}^{2}$. The CKD stage was defined as stage $1+2$ for an eGFR $>60 \mathrm{ml} / \mathrm{min} /$ $1.73 \mathrm{~m}^{2}$, stage 3 for eGFR $30-60 \mathrm{ml} / \mathrm{min} / 1.73 \mathrm{~m}^{2}$, stage 4 for eGFR $15-30 \mathrm{ml} / \mathrm{min} / 1.73 \mathrm{~m}^{2}$, stage 5 for eGFR $<15$ $\mathrm{ml} / \mathrm{min} / 1.73 \mathrm{~m}^{2}$. We used the eGFR basically from the first visit to our hospital up until the performance of lung cancer surgery without any intravenous hydration. As underlying comorbidities other than CKD, cardiovascular and cerebrovascular diseases, chronic obstructive pulmonary disease (COPD), interstitial lung disease (ILD), diabetes mellitus, and the use of steroid or immunosuppressive agents were also evaluated. The criteria used to determine COPD were based on the "Guidelines for the diagnosis and treatment of COPD, 3rd edition" presented by the Japanese Respiratory Society [15]. The length of the operation, intraoperative bleeding, and postoperative complications were also reviewed.

\section{Treatment strategy}

Lobectomy and systemic lymph node dissection (ND2) as a standard surgery were basically performed for lung cancer patients in our institution. The type of surgical procedure, approach, and distribution of lymph node dissection in each patient were based on the characteristics of the tumor and the patient's respiratory function and general condition. Limited resection (segmentectomy or wedge resection) was selected with curative intent in patients indicated to have ground-glass opacity (GGO)-dominant lesions [16]. Limited resection due to comorbidity was selected in cases with a poor respiratory function or other comorbidities, such as cerebral infarction sequelae, dementia, advanced age, or multiple lung cancer tumors [2,3]. CKD alone was not a contraindication for standard surgery if the patients had a good exercise tolerance.

\section{Surgical complications}

Surgical complications were determined from patient medical records after surgery and were then stratified according to the Clavien-Dindo (CD) classification; the CD classification is categorized by surgical complications graded I through $\mathrm{V}$ [17]. If more than one complication occurred in a single patient, the most severe grade was recorded. Grade IIIa or more severe complications were defined as severe complications.

\section{Patient follow-up}

Follow-up was generally based on chest X-ray, a physical examination, and blood chemistry findings, including measurements of carcinoembryonic antigens in serum performed every 3 to 6 months after surgery. Contrastenhanced chest computed tomography (CT), contrast-enhanced brain $\mathrm{CT}$ or magnetic resonance imaging and bone scintigraphy were routinely performed annually during the follow-up period as surveillance for lung cancer recurrence $[2,3]$. The disease-free survival (DFS) was defined as the time interval between the date of pulmonary resection and first recurrence or death due to any cause. The overall survival (OS) was the primary endpoint defined as the time interval between the date of pulmonary resection and death or the last follow-up for surviving patients. The median follow-up time in patients with and without CKD was 51 months (range 5-105 months) and 55 months (range 0-131 months), respectively. Loco-regional recurrence was defined as any recurrence in the ipsilateral lobe, hilum, or mediastinum; pleura dissemination; and malignant pleural effusion in the ipsilateral lung. Distant recurrence was defined as any recurrence in a distinctly different lobe 
of the ipsilateral lung, contralateral mediastinum or hilum and extrathoracic metastatic disease.

\section{Statistical analyses}

The data are expressed as the mean \pm standard deviation or median values. Differences in clinical variables between groups were evaluated using Student's $t$ test, a Chi-square test, or Fisher's exact test. OS and DFS were analyzed by the Kaplan-Meier method, with differences between groups calculated using the log-rank test. To identify the factors related to the OS, a Cox proportional hazards model was used to estimate the level of significance and the relative risk with a $95 \%$ confidence interval (CI). A $p$ value less than 0.05 was considered to indicate statistical significance. All statistical analyses were performed using the JMP Pro 14 software program (SAS Institute, Berkley, CA, USA).

\section{Results}

\section{Characteristics of patients with CKD undergoing lung cancer resection}

From our data, we identified a total of 55 patients with CKD (8\%) who underwent lung cancer resection, including 8 receiving hemodialysis. The numbers of patients with stages 3, 4, and 5 CKD were 43, 3, and 9, respectively. There were no kidney transplant patients operated on for lung cancer in the present study. The clinical characteristics of these patients are summarized in Table 1. Patients with CKD were significantly older than those without CKD. The incidences of male and smoking patients were significantly higher among patients with CKD than among those without CKD. There was a significantly higher incidence of cardiovascular diseases, pulmonary diseases, atherosclerotic diseases, and steroid or immunosuppressive agents use among patients with CKD than among those without CKD. Four patients with CKD used steroid or immunosuppressive agents to control interstitial pneumonitis, rheumatoid arthritis, ANCA-associated glomerulonephritis, and mixed connective tissue disease (MCTD). Patients with CKD showed significantly lower pulmonary function parameters than those without CKD.

\section{Operation-related factors}

Table 2 summarizes the operation-related factors, which differed significantly by the presence of CKD. Operations were significantly shorter in patients with CKD than in those without CKD. Lobectomy was the most common surgical procedure in both groups; however, patients with
CKD were more likely to undergo wedge resection than those without CKD. Furthermore, the proportion of patients who underwent systemic lymph node dissection (ND2) was lower in patients with CKD than those without CKD. Histopathologically, there were about twice as many patients diagnosed with squamous cell carcinoma among patients with CKD as among those without CKD. There were no significant differences in the pathologic stage by the presence of CKD.

\section{Surgical complications}

Table 3 details the incidence of overall postoperative complications. A total of 177 complications were observed in 156 patients. There were no postoperative deaths among the patients with CKD, but four of the patients without CKD died postoperatively. The rate of postoperative complications (grade 1-5) among patients with CKD was $30.9 \%$, which was similar to that in those without CKD (22.6\%, $p=0.16)$. The rate of serious complication (CD grade $\geq 3$ ) among patients with $\mathrm{CKD}$ was $9.1 \%$, which was also similar to that in those without CKD $(7.6 \%, p=0.70)$. The following factors were assessed to determine any potential associations with the development of postoperative complications using a univariate analysis: age, sex, medical history, surgical approach, type of resection, distribution of lymph node dissection, and CKD stage. The results of the univariate analysis are described in Table 4. Patients with a medical history of cardiovascular diseases, hypertension, and COPD were significantly associated with the development of surgical complications, while surgical factors and CKD stages were not associated with the development of surgical complications.

\section{Long-term outcomes}

We analyzed the DFS and OS rates of patients with or without CKD. Figure 1 shows the DFS rates, while Fig. 2 shows the OS rates. The 5-year DFS rates in patients with and without CKD were $60.0 \%$ and $69.7 \%$, respectively, showing no marked intergroup differences $(p=0.06)$. In contrast, the 5-year OS rates in patients with and without CKD were $68.9 \%$ and $80.0 \%$, respectively $(p=0.01)$, showing significant differences. At the time of writing, a total of 20 patients had died in the CKD group: 11 due to primary lung cancer; 2 each due to another cancer, pneumonia and cardiovascular diseases; 1 each due to cerebrovascular disease, multiple organ failure and bleeding due to hematuria. Table 5 summarizes the pattern of recurrences and initial treatment modality. Although the frequencies of local and distant metastasis between the groups were similar, the treatment modality after initial recurrence differed significantly. The rate of receiving 
Table 1 Patient characteristics

\begin{tabular}{|c|c|c|c|}
\hline Patient characteristics & $\begin{array}{l}\text { CKD (\%) } \\
n=55\end{array}$ & $\begin{array}{l}\text { Non-CKD }(\%) \\
n=616\end{array}$ & $p$ value \\
\hline Age (years) & & & $<0.01$ \\
\hline Mean & $73.8 \pm 7.0$ & $67.0 \pm 10.4$ & \\
\hline Range & $59-89$ & $17-88$ & \\
\hline Sex & & & $<0.01$ \\
\hline Male & $44(80 \%)$ & $343(55.7 \%)$ & \\
\hline Female & $11(20 \%)$ & $273(44.3 \%)$ & \\
\hline Smoking status & & & $<0.01$ \\
\hline Never smoker & $11(20 \%)$ & $273(44.3 \%)$ & \\
\hline Smoker & $44(80 \%)$ & $343(55.7 \%)$ & \\
\hline Brinkman index & $813.0 \pm 692.4$ & $588.0 \pm 743.1$ & 0.03 \\
\hline Body mass index & $22.3 \pm 3.0$ & $22.1 \pm 3.0$ & 0.70 \\
\hline \multicolumn{4}{|l|}{ Medical history } \\
\hline Aortic valve disease & $2(3.6 \%)$ & $14(2.3 \%)$ & 0.53 \\
\hline Mitral valve disease & $4(7.3 \%)$ & $12(1.9 \%)$ & 0.01 \\
\hline Congestive heart failure & $7(12.7 \%)$ & $11(1.8 \%)$ & $<0.01$ \\
\hline Hypertension & $20(36.4 \%)$ & $195(31.7 \%)$ & 0.47 \\
\hline Diabetes mellitus & $14(25.5 \%)$ & $94(15.3 \%)$ & 0.05 \\
\hline Cerebral vascular disease & $5(9.1 \%)$ & $38(6.2 \%)$ & 0.40 \\
\hline Atrial fibrillation & $6(10.9 \%)$ & $28(4.5 \%)$ & 0.04 \\
\hline Coronary artery disease & $17(30.9 \%)$ & $28(4.5 \%)$ & $<0.01$ \\
\hline Atherosclerotic disease & $15(27.3 \%)$ & $42(6.8 \%)$ & $<0.01$ \\
\hline COPD & $20(36.4 \%)$ & $133(21.6 \%)$ & 0.01 \\
\hline *ILD & $11(20 \%)$ & $36(5.8 \%)$ & $<0.01$ \\
\hline Malignancy besides LC & $13(23.6 \%)$ & $172(27.9 \%)$ & 0.50 \\
\hline Steroid or immunosuppressive agents use & $4(7.3 \%)$ & $7(1.1 \%)$ & $<0.01$ \\
\hline \multicolumn{4}{|l|}{ Pulmonary function } \\
\hline $\mathrm{VC}(\mathrm{L})$ & $3.0 \pm 0.7$ & $3.2 \pm 0.8$ & 0.07 \\
\hline$\% \mathrm{VC}(\%)$ & $92.4 \pm 13.4$ & $101.1 \pm 14.7$ & $<0.01$ \\
\hline FVC (L) & $2.9 \pm 0.7$ & $3.1 \pm 0.8$ & 0.07 \\
\hline FEV1.0 (L) & $2.1 \pm 0.6$ & $2.3 \pm 0.6$ & 0.01 \\
\hline FEV1.0\% (\%) & $71.7 \pm 10.5$ & $74.2 \pm 9.7$ & 0.08 \\
\hline$\%$ DLco $(\%)$ & $78.1 \pm 21.0$ & $88.2 \pm 20.4$ & $<0.01$ \\
\hline
\end{tabular}

Atherosclerotic disease includes aortic aneurysm and atherosclerosis obliterans

$C K D$ chronic kidney disease, $* I L D$ interstitial lung disease, radiological findings, $C O P D$ chronic obstructive pulmonary disease, $L C$ lung cancer, $V C$ vital capacity, $F V C$ forced vital capacity, FEV forced expiratory volume 1.0 (s), DLco diffusing capacity of the lung carbon monoxide

supportive care was significantly higher among patients with CKD than among those without CKD. A multivariate analysis was performed using the factors selected in the univariate analysis as described in Table 6. CKD was not significant, while the age, sex, histology, postoperative complications, and pathologic stage (pStage) were independent prognostic factors for the survival in lung cancer patients undergoing surgery.

\section{Discussion}

In the present study, we focused on CKD as a comorbidity in lung cancer patients who underwent pulmonary resection. We showed that patients with CKD had a higher frequency of COPD, cardiovascular disease (CVD), and radiological interstitial lung disease (ILD), and a notably lower pulmonary function, than those without CKD, resulting in their receiving limited pulmonary resection and a tendency to have a poor DFS. We also found that patients 
Table 2 Operation-related factors

\begin{tabular}{|c|c|c|c|}
\hline Surgical factors & $\begin{array}{l}\text { CKD (\%) } \\
n=55\end{array}$ & $\begin{array}{l}\text { Non-CKD }(\%) \\
n=616\end{array}$ & $p$ value \\
\hline Approach & & & 0.09 \\
\hline VATS & $23(41.8 \%)$ & $331(53.7 \%)$ & \\
\hline Thoracotomy & $32(58.2 \%)$ & $285(46.3 \%)$ & \\
\hline Type of surgery & & & $<0.01$ \\
\hline Pneumonectomy & 0 & $9(1.5 \%)$ & \\
\hline Lobectomy/bilobectomy & $35(63.6 \%)$ & $464(75.3 \%)$ & \\
\hline Segmentectomy & $8(14.5 \%)$ & $98(15.9 \%)$ & \\
\hline Wedge resection & $12(21.8 \%)$ & $45(7.3 \%)$ & \\
\hline Lymph node dissection & & & $<0.01$ \\
\hline ND0 & $21(38.2 \%)$ & $142(23.1 \%)$ & \\
\hline ND1 & $14(25.5 \%)$ & $88(14.3 \%)$ & \\
\hline ND2 & $20(36.3 \%)$ & $386(62.6 \%)$ & \\
\hline Length of operation (min) & $195.8 \pm 72.4$ & $225.0 \pm 83.7$ & 0.01 \\
\hline Intraoperative bleeding $(\mathrm{g})$ & $171.3 \pm 197.6$ & $223.7 \pm 480.3$ & 0.42 \\
\hline Pathologic stage of lung cancer & & & 0.72 \\
\hline IA & $33(60 \%)$ & $363(58.9 \%)$ & \\
\hline IB & $13(23.6 \%)$ & $115(18.7 \%)$ & \\
\hline IIA & $4(7.3 \%)$ & $55(8.9 \%)$ & \\
\hline IIB & $1(1.8 \%)$ & $29(4.7 \%)$ & \\
\hline IIIA & $4(7.3 \%)$ & $42(6.8 \%)$ & \\
\hline IIIB/V & 0 & $12(1.9 \%)$ & \\
\hline Histologic type of lung cancer & & & $<0.01$ \\
\hline Adenocarcinoma & $35(63.6 \%)$ & $491(79.7 \%)$ & \\
\hline Adenosquamous cell carcinoma & $1(1.8 \%)$ & $3(0.5 \%)$ & \\
\hline Squamous cell carcinoma & $19(34.5 \%)$ & $95(15.4 \%)$ & \\
\hline Other & 0 & $27(4.4 \%)$ & \\
\hline
\end{tabular}

$C K D$ chronic kidney disease, VATS video-assisted thoracoscopic surgery, $N D$ lymph node dissection

with CKD had a poorer OS than those without CKD. To our knowledge, this is the first study to evaluate the influence of the renal function on the clinical features and survival outcomes of patients who underwent lung cancer surgery.

The influence of CKD on the lung cancer survival has not been clarified. Few studies have evaluated the outcomes of lung cancer among patients with CKD, and the association between CKD and the prognosis of lung cancer is controversial $[12,18,19]$. In a retrospective study of 107 lung cancer patients with CKD, Patel et al. [18] reported that CKD did not have influence the clinical course or survival compared with non-CKD. However, some authors have conversely reported that CKD had a negative influence on the prognosis of lung cancer. Cenik et al. [19] found that the renal function might be inversely associated with the survival in 298 patients with stage IV non-small cell lung cancer (NSCLC). According to a nationwide
Taiwan-based cohort study, CKD was independently associated with increased mortality in lung cancer patients [12]. The presence of CKD may limit the therapeutic options available to lung cancer patients, resulting in suboptimal management. However, no study has evaluated lung cancer patients with CKD limited to surgical cases. Therefore, to our knowledge, this is the first report that CKD has a negative influence on the OS in patients undergoing lung cancer surgery.

CKD is associated with other comorbid diseases. The present study showed that patients with CKD were older and with a higher incidence of smoking than those without $\mathrm{CKD}$, and the frequencies of coexisting CVD, COPD, and ILD were also higher among patients with CKD than among those without it. Regarding accompanying disorders, the pulmonary function was notably lower in patients with CKD than in those without it, resulting in the CKD patients receiving limited pulmonary resection. An 
Table 3 Postoperative complications

\begin{tabular}{lll}
\hline Postoperative complications & $\begin{array}{l}\text { CKD } \\
n=55\end{array}$ & $\begin{array}{l}\text { Non-CKD } \\
n=616\end{array}$ \\
\hline Postoperative arrhythmia & $6(10.9 \%)$ & $50(8.1 \%)$ \\
Myocardial infarction/angina & $1(1.8 \%)$ & $4(0.6 \%)$ \\
Bronchopleural fistula & $1(1.8 \%)$ & $2(0.3 \%)$ \\
Prolonged air leak & $3(5.5 \%)$ & $35(5.7 \%)$ \\
Pyothorax & 0 & $10(1.6 \%)$ \\
Pneumonia & $4(7.3 \%)$ & $8(1.3 \%)$ \\
Chylothorax & $1(1.8 \%)$ & $8(1.3 \%)$ \\
Recurrent nerve palsy & $1(1.8 \%)$ & $7(1.1 \%)$ \\
Delirium & 0 & $4(0.6 \%)$ \\
Postoperative bleeding & 0 & $6(1.0 \%)$ \\
Wound infection & $1(1.8 \%)$ & $4(0.6 \%)$ \\
Urinary tract infection & $1(1.8 \%)$ & $2(0.3 \%)$ \\
Acute exacerbation of IP & 0 & $1(0.2 \%)$ \\
Other complications & $1(1.8 \%)$ & $16(2.6 \%)$ \\
Clavien-Dindo classification & & $86(14.0 \%)$ \\
Grade II & $11(20.0 \%)$ & $47(7.6 \%)$ \\
Grade IIIa or more & $5(9.1 \%)$ & \\
\hline CKD chro kin) & &
\end{tabular}

$C K D$ chronic kidney disease, $I P$ interstitial pneumonia

advanced age, smoking history, hypertension, and diabetes mellitus are well known to be important risk factors for CKD [20]. CKD has many characteristics in common with COPD, such as the dependence of the patient prognosis on CVD [21]. However, little is known regarding the association between ILD and CKD. We speculated that there were two points to note regarding the association between CKD and ILD. First, there are a number of systemic autoimmune diseases that are related to both CKD and ILD, including rheumatism, systemic lupus erythematosus, and MCTD. Second, there is an association between idiopathic pulmonary fibrosis (IPF) and CKD. A recent retrospective study on 124 patients with IPF and 61 patients with COPD as the control disease reported that CKD was highly prevalent among patients with IPF as well as COPD [22]. Recent epidemiologic studies have shown that patients with IPF have an increased risk of hypertension, diabetes mellitus, and CVD, which are closely associated with CKD [23]. In addition, IPF, COPD, and CKD share some common clinical risk factors, such as an advanced age and smoking history, suggesting a correlation among them. It is reported that smoking is associated with a higher relative risk of lung squamous cell carcinoma than of adenocarcinoma [24]. Moreover, several studies reported that the most frequently occurring lung cancer type in patients with IPF is squamous cell carcinoma [25, 26]. As a result of these factors related to $\mathrm{CKD}$, the frequency of squamous cell carcinoma was higher in patients with $\mathrm{CKD}$ than in those without CKD.

To our knowledge, the present study was the first to analyze the association between CKD and postoperative complications of pulmonary resection. Several studies have reported that renal dysfunction increases the risk of anastomotic leakage following gastrointestinal surgery [7, 8]. Furthermore, the frequencies of postoperative cardiovascular events have been reported to also be higher in patients with CKD than in those without CKD. In contrast, CKD did not affect the rate of postoperative complications of lung cancer surgery as shown in the present study. Postoperative arrhythmia was the most common complication, regardless of the presence of CKD. The rates of bronchopleural fistula and prolonged air leakage were also similar between the two groups. Because the frequencies of coexisting COPD and ILD were higher in patients with CKD than in those without it, the lung tissues of CKD patients tended to be emphysematous. We proactively control air leakage using a free subcutaneous fat pad during surgery, which is effective in reducing the duration of air leakage and chest tube drainage [27]. These preventive procedures for air leakage are also considered effective in patients with CKD. However, pneumonia after pulmonary resection was more frequently observed in patients with CKD $(n=4,7.3 \%)$ than in those without CKD $(n=8$, $1.3 \%, p<0.01)$. Several mechanisms potentially linking CKD and pneumonia have been proposed. Immune response deficiency is a common occurrence, and the humoral immune defense is often compromised in patients with CKD [28, 29]. In addition, the present study showed that comorbidity of hypertension, COPD, and CVD was associated with the occurrence of postoperative complications. Thoracic surgeons should be alert for postoperative complications when these patients undergo pulmonary resection.

Our main concern when treating patients with CKD was whether or not less invasive pulmonary resection and lymph node dissection performed due to their worse clinical characteristics affects the oncological outcome. Our findings that the DFS did not differ significantly between the two groups mean that our treatment strategy is reasonable and suggests that lymph node dissection may be lessened in the current lung cancer surgery strategy. Recently, several studies demonstrated that selective lymph node dissection is not inferior to standard systematic lymph node dissection in terms of the postoperative prognosis in lung cancer surgery $[30,31]$. Furthermore, the pattern of recurrence between the two groups was similar in the present study. Thus, thoracic surgeons should carefully consider the level of lymph node dissection using an intraoperative frozen histological examination and lymph node sampling as much as possible in patients with CKD. 
Table 4 A univariate analysis of the factors associated with the postoperative complications

\begin{tabular}{|c|c|c|c|}
\hline & $\begin{array}{l}\text { Complication } \\
(n=17)\end{array}$ & $\begin{array}{l}\text { Non-complication } \\
(n=38)\end{array}$ & $p$ value \\
\hline Sex & & & 0.77 \\
\hline Male/female & $14 / 3$ & $30 / 8$ & \\
\hline Age & $75.9 \pm 7.4$ & $72.9 \pm 7.2$ & 0.16 \\
\hline \multicolumn{4}{|l|}{ Comorbidity other than CKD } \\
\hline Cardiovascular disease $^{\mathrm{a}}$ & $12(70.6 \%)$ & $12(31.6 \%)$ & $<0.01$ \\
\hline Hypertension & $11(64.7 \%)$ & $9(23.7 \%)$ & $<0.01$ \\
\hline Diabetes mellitus & $4(23.5 \%)$ & $10(26.3 \%)$ & 0.83 \\
\hline Atherosclerosis & $6(35.3 \%)$ & $9(23.7 \%)$ & 0.37 \\
\hline COPD & $10(58.8 \%)$ & $10(26.3 \%)$ & 0.02 \\
\hline $\mathrm{ILD}^{\mathrm{b}}$ & $4(23.5 \%)$ & $7(18.4 \%)$ & 0.66 \\
\hline Malignancy besides LC & $5(29.4 \%)$ & $8(21.1 \%)$ & 0.50 \\
\hline Length of operation (min) & $210.3 \pm 81.3$ & $189.4 \pm 68.1$ & 0.33 \\
\hline Intraoperative bleeding (g) & $181.2 \pm 192.4$ & $166.9 \pm 202.3$ & 0.81 \\
\hline Surgical approach & & & 0.51 \\
\hline Thoracotomy/VATS & $11 / 6$ & $21 / 17$ & \\
\hline Type of resection & & & 0.44 \\
\hline Lobectomy/segmentectomy/WWR & $10 / 4 / 3$ & $25 / 4 / 9$ & \\
\hline Lymph node dissection & & & 0.76 \\
\hline ND0/ND1/ND2 & $7 / 5 / 5$ & $14 / 9 / 15$ & \\
\hline CKD stage $3 / 4+5$ & $14 / 3$ & $29 / 9$ & 0.62 \\
\hline
\end{tabular}

Atherosclerotic disease includes aortic aneurysm and atherosclerosis obliterans

$C K D$ chronic kidney disease, $C O P D$ chronic obstructive pulmonary disease, VATS video-assisted thoracoscopic surgery, $L C$ lung cancer, $W W R$ wide wedge resection, $N D$ lymph node dissection

${ }^{\mathrm{a}}$ Cardiovascular disease includes aortic valve disease, mitral valve disease, chronic heart failure, coronary artery disease, and atrial fibrillation

${ }^{\mathrm{b}} \mathrm{ILD}$, interstitial lung disease, radiological findings

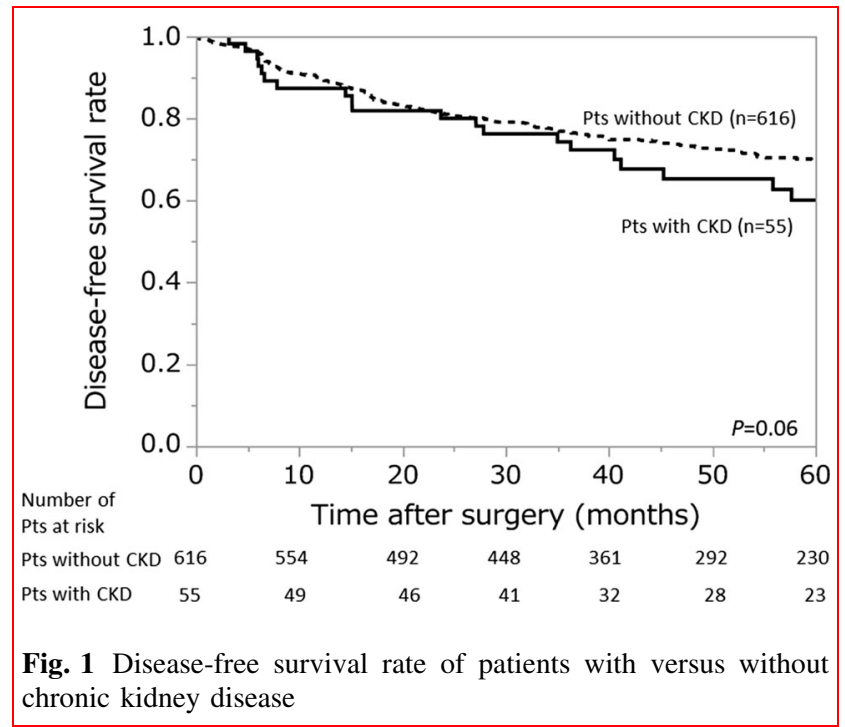

However, the OS differed significantly between the two groups. It is speculated that patients with CKD were not physically fit to receive systemic chemotherapy, which

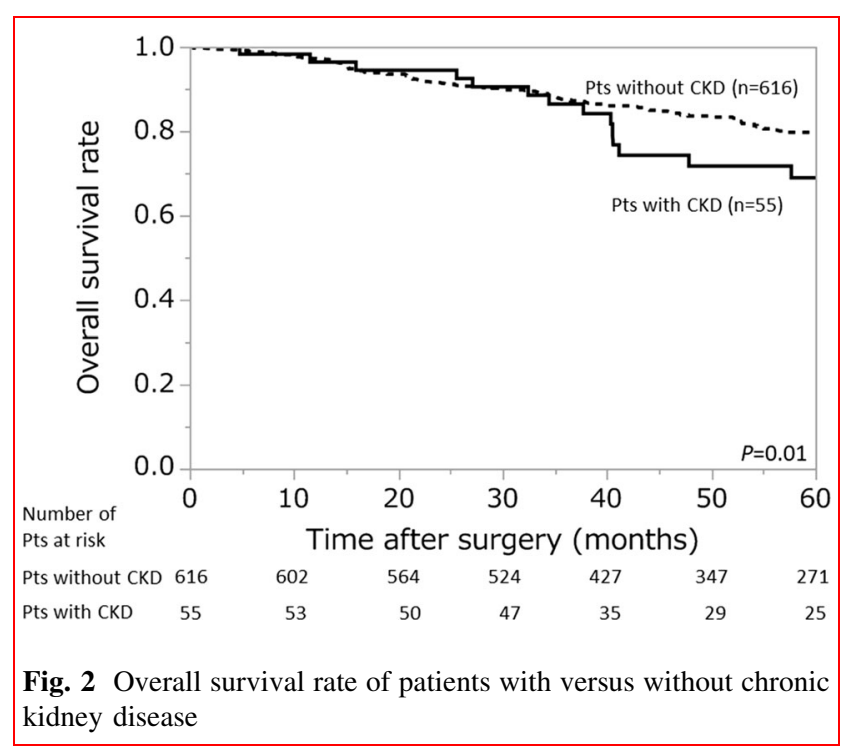

subsequently reduced their OS. Of note, cytotoxic chemotherapy has renal toxicity and so was generally not 
Table 5 Pattern of recurrences and treatment modality

\begin{tabular}{lll}
\hline & $\begin{array}{l}\text { CKD }(\%) \\
n=15\end{array}$ & $\begin{array}{l}\text { Non-CKD (\%) } \\
n=141\end{array}$ \\
\hline Recurrent site & & $47(33.3 \%)$ \\
Local & $5(33.3 \%)$ & $92(65.2 \%)$ \\
Distant & $10(66.7 \%)$ & $2(1.5 \%)$ \\
Unknown & 0 & \\
Treatment modality for initial recurrence & & $23(16.3 \%)$ \\
Supportive care & $6(40 \%)$ & $18(12.8 \%)$ \\
Surgery & $2(13.3 \%)$ & $28(20.0 \%)$ \\
Radiation therapy & $3(20 \%)$ & $63(44.7 \%)$ \\
Systemic chemotherapy & $3(20 \%)$ & $9(6.4 \%)$ \\
Unknown & $1(6.7 \%)$ & 0.03 \\
Administration of molecular-targeted therapy & & $34(24.1 \%)$ \\
eGFR-TKIs & $1(6.7 \%)$ & $2(1.4 \%)$ \\
ALK-inhibitors & $1(6.7 \%)$ & $1(0.7 \%)$ \\
ICIs & 0 & 0.95 \\
\hline
\end{tabular}

$C K D$, chronic kidney disease, eGFR-TKI epidermal growth factor receptor tyrosine kinase inhibitors, $A L K$ anaplastic lymphoma kinase, ICI immune checkpoint inhibitors

Table 6 Univariate and multivariate analyses of prognostic factors for patients undergoing lung cancer surgery

\begin{tabular}{|c|c|c|c|c|}
\hline \multirow[t]{2}{*}{ Clinical variable } & \multicolumn{2}{|l|}{ Univariate analysis } & \multicolumn{2}{|l|}{ Multivariate analysis } \\
\hline & Odds ratio $(95 \% \mathrm{CI})$ & $p$ value & Odds ratio $(95 \% \mathrm{CI})$ & $p$ value \\
\hline Age $(<70$ vs. $70 \leq)$ & $0.410(0.290-0.572)$ & $<0.01$ & $0.430(0.300-0.608)$ & $<0.01$ \\
\hline Sex (male vs. female) & $3.293(2.249-4.975)$ & $<0.01$ & $2.776(1.592-4.885)$ & $<0.01$ \\
\hline Smoking (smoker vs. never smoker) & $2.567(1.783-3.786)$ & $<0.01$ & $0.776(0.449-1.368)$ & 0.37 \\
\hline HT (yes vs. no) & $1.369(0.972-1.907)$ & 0.07 & & \\
\hline DM (yes vs. no) & $1.577(1.059-2.287)$ & 0.03 & $0.972(0.644-1.430)$ & 0.89 \\
\hline COPD (yes vs. no) & $1.880(1.317-2.644)$ & $<0.01$ & $0.953(0.646-1.396)$ & 0.80 \\
\hline Malignancy besides LC (yes vs. no) & $1.306(0.916-1.835)$ & 0.14 & & \\
\hline CKD (CKD vs. non-CKD) & $1.821(1.103-2.850)$ & 0.02 & $1.418(0.846-2.264)$ & 0.16 \\
\hline Approach (thoracotomy vs. VATS) & $2.282(1.620-3.262)$ & $<0.01$ & $1.379(0.953-2.018)$ & 0.09 \\
\hline Type of surgery (lobectomy more vs. sublobar) & $1.102(0.755-1.657)$ & 0.62 & & \\
\hline Histology (Ad vs. non-Ad) & $0.337(0.244-0.471)$ & $<0.01$ & $0.654(0.456-0.941)$ & 0.02 \\
\hline Postoperative complications (yes vs. no) & $2.152(1.528-3.000)$ & $<0.01$ & $1.656(1.163-2.336)$ & $<0.01$ \\
\hline pStage (II, III, IV vs. I) & $3.474(2.505-4.798)$ & $<0.01$ & $2.763(1.933-3.942)$ & $<0.01$ \\
\hline
\end{tabular}

$H T$ hypertension, $D M$ diabetes mellitus, $C O P D$ chronic obstructive pulmonary disease, $L C$ lung cancer, $C K D$ chronic kidney disease, VATS video-assisted thoracoscopic surgery, Ad adenocarcinoma, pStage pathologic stage

introduced for patients with CKD. Regarding the treatment for recurrent disease, the proportion of patients who received supportive care was significantly higher in those with CKD than in those without CKD. However, our multivariate analysis showed that CKD was not significant, while age, sex, histology, postoperative complications, and pStage were independent prognostic factors for the survival in patients undergoing lung cancer surgery. The presumed reason for this is as follows: CKD group demonstrated a poor prognosis according to a univariate analysis because the CKD group was associated with poor prognostic factors in their background. As CKD alone was not an independent prognostic factor, the most suitable treatment for patients with CKD determined after taking clinical factors other 
than CKD into consideration might result in a survival outcome not inferior to that in patients without CKD. Based on our findings, selecting the appropriate extent of pulmonary resection and lymph node dissection while balancing radicality and surgical stress is important for treating patients with CKD.

Several limitations associated with the present study warrant mention. First, we had a small sample size and our data were necessarily derived from a single institution, limiting the power of our statistical findings. Second, because of the long duration of this study, the diagnostic modalities changed with the introduction of positron emission tomography. Advancements in radiologic examination techniques might therefore have affected the patient selection. Third, the classification of patients into CKD stages was based on the preoperative creatinine level without consideration of chronicity. Finally, the retrospective design introduces inevitable selection bias. Further studies are needed to achieve a more detailed conclusion.

In conclusion, we herein report for the first time that CKD is associated with a poor OS among patients who undergo lung cancer surgery. The suboptimal treatment choice for recurrent disease might explain this result in part. Because patients with CKD are limited in their choice of systemic treatment, surgery remains a mainstay for the treatment of lung cancer in this population. As patients with CKD tend to have a poor respiratory function, thoracic surgeons should carefully select the type of resection to achieve a balance between the therapeutic benefit and invasiveness in these patients.

Acknowledgements This article was supported by advanced clinical epidemiology investigators in Osaka University Graduate School of Medicine and Osaka University budget request grant for research center of medical data science.

Funding Open access funding provided by Lund University.

\section{Compliance with ethical standards}

Conflict of interest The authors declare no conflicts of interest in association with the present study.

Open Access This article is distributed under the terms of the Creative Commons Attribution 4.0 International License (http://crea tivecommons.org/licenses/by/4.0/), which permits unrestricted use, distribution, and reproduction in any medium, provided you give appropriate credit to the original author(s) and the source, provide a link to the Creative Commons license, and indicate if changes were made.

\section{References}

1. Siegel R, Naishadham D, Jemal A (2013) Cancer statistics 2013. CA Cancer J Clin 63:11-30
2. Kanzaki R, Inoue M, Minami M et al (2017) Outcomes of lung cancer surgery in patients with coronary artery disease; a decade of experience at a single institution. Surg Today 47:27-34

3. Kanzaki R, Kimura T, Kawamura T et al (2017) Outcomes of surgery for lung cancer in patients with atrial fibrillation as a preoperative comorbidity: a decade of experience at a single institution in Japan. Surg Today 47:795-801

4. National Kidney Foundation (2002) K/DOQI clinical practice guidelines for chronic kidney disease: evaluation, classification, and stratification. Accessed online April 2002. http://www.kid ney.org/professionals/doqi/kdoqi/toc.htm

5. Cuckovic C, Djukanovic L, Jankovic S et al (1996) Malignant tumors in hemodialysis patients. Nephron 73:710-712

6. Inamoto H, Ozaki R, Matsuzaki T et al (1991) Incidence and mortality patterns of malignancy and factors affecting the risk of malignancy in dialysis patients. Nephron 59:611-617

7. Matusmoto S, Takayama T, Wakatsuki K et al (2014) Short-term and long-term outcomes after gastrectomy for gastric cancer in patients with chronic kidney disease. World J Surg 38:1453-1460. https://doi.org/10.1007/s00268-013-2436-4

8. Nozawa H, Kitayama J, Sunami E et al (2012) Impact of chronic kidney disease on outcomes of surgical resection for primary colorectal cancer: a retrospective cohort review. Dis Colon Rectum 55:94856

9. Antoniak D, Are C, Vokoun C et al (2018) The relationship between age and chronic kidney disease in patients undergoing pancreatic resection. J Gastrointest Surg 22:1376-1384

10. Lee CH, Hsieh SY, Lin JL et al (2013) Hepatocellular carcinoma in patients with chronic kidney disease. World J Gastroenterol 19:2466-2472

11. Homma A, Hayashi R, Kawabata K et al (2016) Association of impaired renal function and poor prognosis in oropharyngeal squamous cell carcinoma. Head Neck 38:1495-1500

12. Wei YF, Chen JY, Lee HS et al (2018) Association of chronic kidney disease with mortality risk in patients with lung cancer: a nationwide Taiwan population-based cohort study. BMJ Open 8:e019661. https://doi.org/10.1136/bmjopen-2017-019661

13. Goldstraw P, Crowley J, Chansky K et al (2007) The IASLC Lung Cancer Staging Project: proposals for the revision of the TNM stage groupings in the forthcoming (seventh) edition of the TNM classification of malignant tumours. $J$ Thorac Oncol 2:706-714

14. Japanese nephrology society (2012) Clinical practice guidebook for diagnosis and treatment of chronic kidney disease 2012. Nihon Jinzo Gakkai Shi 54:1034-1191

15. Committee for the Third Edition of the COPD Guidelines of The Japanese Respiratory Society (2009) Guidelines for the diagnosis and treatment of COPD (chronic obstructive disease), 3rd edn. Nihon Kokyuki Gakkai Zasshi 2009; Suppl Copd

16. Inoue M, Minami M, Sawabata N et al (2010) Surgical treatment strategy for small-sized, non-small cell lung cancer: a proposal algorithm for limited resection. J Jpn Assoc Chest Surg 24:1004-1010

17. Katayama H, Kurokawa Y, Nakamura K et al (2016) Extended Clavien-Dindo classification of surgical complications: Japan Clinical Oncology Group postoperative complications criteria. Surg Today 46:668-685

18. Patel P, Henry LL, Ganti AK et al (2004) Clinical course of lung cancer in patients with chronic kidney disease. Lung Cancer 43:297-300

19. Kutluk Cenik B, Sun H, Gerber DE (2013) Impact of renal function on treatment options and outcomes in advanced nonsmall cell lung cancer. Lung Cancer 80:326-332

20. James MT, Hemmelgarn BR, Tonelli M (2010) Early recognition and prevention of chronic kidney disease. Lancet 375:1296-1309 
21. Yoshizawa T, Okada K, Furuichi S et al (2015) Prevalence of chronic kidney diseases in patients with chronic obstructive pulmonary disease: assessment based on glomerular filtration rate estimated from creatinine and cystatin $\mathrm{C}$ levels. Int $\mathrm{J}$ Chron Obstruct Pulmon Dis 10:1283-1289

22. Ikezoe K, Handa T, Tanizawa K et al (2017) Chronic kidney disease predicts survival in patients with idiopathic pulmonary fibrosis. Respiration 94:346-354

23. Nathan SD, Basavaraj A, Reichner C et al (2010) Prevalence and impact of coronary artery disease in idiopathic pulmonary fibrosis. Respir Med 104:1035-1041

24. Egawa H, Furukawa K, Preston D et al (2012) Radiation and smoking effects on lung cancer incidence by histological types among atomic bomb survivors. Radiat Res 178:191-201

25. Karampitsakos T, Tzilas V, Tringidou R et al (2017) Lung cancer in patients with idiopathic pulmonary fibrosis. Pulm Pharmacol Ther 45:1-10

26. Tomassettis S, Gurioli C, Ryu JH et al (2015) The impact of lung cancer on survival of idiopathic pulmonary fibrosis. Chest 147:157-164

27. Shintani Y, Inoue M, Funaki S et al (2015) Clinical usefulness of free subcutaneous fat pad for reduction of intraoperative air leakage during thoracoscopic pulmonary resection in lung cancer cases. Surg Endosc 29:2910-2913

28. James MT, Quan H, Tonelli M et al (2009) CKD and risk of hospitalization and death with pneumonia. Am J Kidney Dis 54:24-32

29. Ishigami J, Grams ME, Chang AR et al (2017) CKD and risk for hospitalization with infection: the atherosclerosis risk in communities (ARIC) study. Am J Kidney Dis 69:752-761

30. Adachi H, Sakamaki K, Nishii T et al (2017) Lobe-specific lymph node dissection as a standard procedure in surgery for non-small cell lung cancer: a propensity score matching study. J Thorac Oncol 12:85-93

31. Hishida T, Miyaoka E, Yokoi K et al (2016) Lobe-specific nodal dissection for clinical stage I and II NSCLC: Japanese multiinstitutional retrospective study using a propensity score analysis. J Thorac Oncol 11:1529-1537

Publisher's Note Springer Nature remains neutral with regard to jurisdictional claims in published maps and institutional affiliations. 